\title{
A Rare Case of Acute Axonal Motor Neuropathy in a Puerperal Woman
}

\author{
${ }^{1}$ Ashwini Nayak, ${ }^{2} \mathrm{~N}$ Sundari, ${ }^{3}$ Asha Swarup, ${ }^{4}$ Jyothi Girish
}

\begin{abstract}
We report the case of a puerperal woman who presented to us with sepsis, multiorgan dysfunction and motor weakness of both lower limbs. On detailed evaluation, patient was found to have axonal neuropathy establishing the diagnosis of critical illness polyneuropathy (CIP). A high index of suspicion is required to arrive at the diagnosis as this condition is not only associated with high mortality and morbidity rates but also can affect the quality of life of the individual in the long-term. This case has been reported to highlight the importance of recognition of this common, but rarely diagnosed condition as it can help us to portend the prognosis.
\end{abstract}

Keywords: Axonal polyneuropathy, Critical care, Sepsis.

How to cite this article: Nayak A, Sundari N, Swarup A, Girish J. A Rare Case of Acute Axonal Motor Neuropathy in a Puerperal Woman. J South Asian Feder Obst Gynae 2015; 7(1):48-49.

Source of support: Nil

Conflict of interest: None

\section{INTRODUCTION}

Critical illness polyneuropathy (CIP) is an acute disorder of neuromuscular system affecting severely ill patients. Complex pathogenetic mechanisms have been hypothesized involving metabolic, inflammatory, and bioenergetic alterations supporting microvascular changes in peripheral nerves, though its etiology remains unclear. ${ }^{1}$ Usually, the motor nerve fibers are affected. Sensory fibres and cranial nerves are generally preserved. ${ }^{2}$ Multiorgan failure, sepsis, and critical illness polyneuropathy have a mortality rate of $50 \% .{ }^{3}$ We describe a patient who developed sepsis and went into multiorgan dysfunction with lower limb weakness following delivery which prompted us to diagnose CIP.

\footnotetext{
${ }^{1}$ Associate Professor, ${ }^{2-4}$ Professor

${ }^{1-4}$ Department of Obstetrics and Gynecology, MS Ramaiah Medical College, Bengaluru, Karnataka, India
}

Corresponding Author: Ashwini Nayak, Associate Professor Department of Obstetrics and Gynecology, MS Ramaiah Medical College, Bengaluru, Karnataka, India, Phone: 08028392953, e-mail: ms.drashwini@rediffmail.com

\section{CASE REPORT}

A 25-year-old puerperal lady presented with acute onset of pain, swelling and weakness of lower limbs for 3 days. The patient was a Para 1 Living 1 Abortion 2 and had underwent an emergency cesarean section for fetal distress 3 days prior to admission. Patient had history of fever 1 week prior to delivery. She had no previous hospitalizations or significant medical illness in the past. On examination, she was conscious and well oriented. She was febrile with a temperature of $100^{\circ} \mathrm{F}$. There was pedal edema, pallor and icterus. Pulse was $92 /$ minute and blood pressure (BP) was 140/80 mm Hg. Per abdominal examination revealed an involuting uterus. Examination of the lower limbs showed that both limbs had pitting pedal edema. Peripheral pulses were felt in both lower limbs. There was hypotonia of the lower limbs (hip flexors, extensors $1 / 5$, quadriceps $2 / 5$, distally $3 / 5$ ). Deep tendon reflexes were absent. There was no sensory deficit. The routine urine examination showed no sugar or protein. Her hemoglobin $(\mathrm{Hb})$ was $9.3 \mathrm{gm} / \mathrm{d} 1$, white blood cell (WBC) was $19,600 / \mathrm{mm}^{3}$ and platelets was $1,20,000 /$ $\mathrm{mm}^{3}$. The serum sodium was $134 \mathrm{mEq} / 1$ and potassium was $8 \mathrm{mEq} / \mathrm{l}$. The hyperkalemia was corrected by parenteral infusion of calcium gluconate and $25 \%$ dextrose and insulin. The blood urea was $30 \mathrm{mg} / \mathrm{dl}$ and creatinine was $2.5 \mathrm{mg} / \mathrm{dl}$. The serum bilirubin was $7.9 \mathrm{mg} / \mathrm{d} 1$, direct bilirubin was $7.26 \mathrm{mg} / \mathrm{d} 1$, serum glutamic oxaloacetic transaminase (SGOT) was $40 \mathrm{U} / \mathrm{I}$ and serum glutamic pyruvic transaminase (SGPT) was $35 \mathrm{U} / \mathrm{I}$. Prothrombin time was 18.4 seconds and activated partial thromboplastin time was 32.5 seconds and INR was 1.28. She was treated with parenteral piperacillin-tazobactum and metronidazole. Nerve conduction study was done and the electrophysiological studies (EPS) showed marked decrease of compound muscle action potentials in bilateral common peroneal nerve. The sensory nerve action potentials were normal in the upper and lower limbs. The findings were suggestive of bilateral common peroneal nerve axonal motor neuropathy. Venous Doppler of the lower limbs was found to be normal. Ultrasound of the abdomen showed moderate ascites and right pleural effusion and involuting uterus. With supportive treatment patient recovered and she was discharged 15 days later with the advice to continue exercises at home. 
The patient has had regular follow-up for 3 years after discharge, and she has regained near normal power in her lower limbs.

\section{DISCUSSION}

By definition, CIP is an acute reversible neuropathy that develops during the treatment of critically ill patients. ${ }^{4}$ This newly acquired neuromuscular cause of weakness has been found in $46 \%$ (95\% confidence interval $43-49 \%$ ) critically ill patients with sepsis, multi-organ failure or prolonged mechanical ventilation. ${ }^{5}$ Our patient presented with sepsis and multiorgan failure. Laboratory investigations are nonspecific. ${ }^{6}$ Electrophysiologic findings are those of a pure axonal degeneration, affecting motor than sensory fibers. ${ }^{7}$ In this patient, the electrophysiological studies showed marked decrease of compound muscle action potentials in bilateral common peroneal nerve and normal sensory nerve action potentials. Sepsis, hyperglycemia and decreased serum albumin concentrations are associated with decrease in peripheral nerve function. ${ }^{8}$ The serum albumin in this patient was low $(1.3 \mathrm{~g} / \mathrm{dl})$. Treatment is supportive, initially consisting of aggressive pulmonary hygiene and prevention of secondary complications of immobility, such as skin breakdown, deep venous thrombosis and superimposed compressive neuropathies. ${ }^{7}$ Our patient recovered with symptomatic treatment. The recovery in patients with CIP is spontaneous but gradual. ${ }^{9}$ Critical illness polyneuropathy or critical illness myopathy is associated with increased intensive care unit (ICU) and hospital stays and elevated mortality rates, although other data suggest that patient selection may partially explain this. ${ }^{10}$ Our patient is symptomatically better on follow-up and regained normal power in both her lower limbs.

\section{CONCLUSION}

This case has been presented to highlight the importance of considering this possibility in patients who present with weakness of lower limbs in the presence of severe sepsis as this can help us to prognosticate the disease.

\section{REFERENCES}

1. Latronico N, Peli E, Botteri M. Critical illness myopathy and neuropathy. Curr Opin Crit Care 2005;11(2):126-132.

2. Gattia G, Grassib P, Silvestric L, Zingonea B. Critical illness polyneuropathy: regression following cardiac operation. Interact Cardiovasc Thorac Surg 2007;6(3):419-420.

3. Gutmann L, Gutmann L. Critical illness neuropathy and myopathy. Arch Neurol 1999;56(5):527-528.

4. Khilnani GC, Bansal R. Neuromuscular weakness in critically Ill. JAPI 2004 Feb;52:131-136.

5. Stevens RD, Dowdy DW, Michaels RK, Mendez-Tellez PA, Pronovost PJ, Needham DM. Nueromuscular dysfunction acquired in critical careillness. IntCareMed 2007;33(1):1876-1891.

6. Gurjar M, Azim A, Baronia AK, Poddar B. Facial nerve involvement in critical illness polyneuropathy. Ind J Anaesth 2010 Sep-Oct;54(5):472-474.

7. Vijayan J, Alexander M. Critical illness neuropathy. Ind J Crit Care Med 2005;9(1):32-34.

8. Witt NJ, Zochodne DW, Bolton CF, et al. Peripheral nerve function in sepsis and multiple organ failure. Chest 1991;99(1): 176-184.

9. Khilnani GC, Bansal R, Malhotra OP, Bhatia M. Critical illness polyneuropathy: how often do we diagnose it? Ind J Chest Dis Allied Sci 2003;45:209-213.

10. Hermans G, Jonghe BD, Bruyninckx F, den Berghe GV. Clinical review: critical illness polyneuropathy and myopathy. Critical Care 2008;12(6):238. 